\title{
Sustainability in Biodiversity Software Development: More financing or better practices?
}

\author{
Matthew J Yoder ${ }^{\ddagger}$, Michael B Twidale§, Andrea K Thomerl \\ ‡ University of Illinois, Illinois Natural History Survey, Champaign, United States of America \\ $\S$ University of Illinois, Urbana-Champaign, Graduate School of Library and Information Science, Champaign, United States of \\ America \\ | School of Information, University of Michigan, Ann Arbor, United States of America
}

\section{Corresponding author: Matthew J Yoder (diapriid@gmail.com)}

Received: 14 Aug 2017| Published: 14 Aug 2017

Citation: Yoder M, Twidale M, Thomer A (2017) Sustainability in Biodiversity Software Development: More financing or better practices? Proceedings of TDWG 1: e20283.

https://doi.org/10.3897/tdwgproceedings.1.20283

\begin{abstract}
The Species File Group is a small, endowed team who seeks to provided software tools and related technical resources to communities dependent on biodiversity informatics. We will present an overview of the technical products and services the group provides, including software development, data migration, data hosting and data mobilization services. Given the modest size of the Species File group, we have identified our limitations and future capability needs. These include scaling user and technical support services, increasing capability to migrate bespoke legacy data, more rapidly developing rich user interfaces, increasing the speed and scope of our data processing, and providing archival services.

Our vision leads us to conclude that different types of applications must be put into production and sustained for varying durations of time; plotting the various kinds of software tools needed on a temporal axis creates a picture of how software ecosystems might evolve, and further helps us to identify where we might focus increased funding. Currently however, there are important issues with software sustainability that, if addressed now, could multiply the utility and value of existing community software investments. These include: (1) architecting a software ecosystem that lowers barriers to entry for those willing to contribute to all aspects of a project, (2) improving the modularity of software
\end{abstract}


components, (3) educating scientists in "carpentry" related concepts, (4) increasing the engagement of experts from fields such as human-computer interaction, user-interface (UI) and user-experience (UX) design, and (5) narrowing the iteration time between new data standards and the new software that employs them in order to more rapidly improve both.

\section{Keywords}

Biodiversity Software, Sustainability, Financing, Open Source

\section{Presenting author}

Matthew Yoder

\section{Acknowledgements}

Jim Beach provided feedback that significantly improved the abstract, and that helped to focus the scope of the presentation. 